This document is the accepted manuscript version of the following article:

Römmeler, A., Zolliker, P., Neuenschwander, J., van Gemmeren, V., Weder, M., \& Dual, J.

(2020). Air coupled ultrasonic inspection with Lamb waves in plates showing mode conversion.

U1trasonics, 100, 105984 (12 pp.). https://doi.org/10.1016/j.u1tras.2019.105984

This manuscript version is made available under the CC-BY-NC-ND 4.0 1icense

http: //creativecommons.org/1icenses/by-nc-nd/4.0/

\title{
Air coupled ultrasonic inspection with Lamb waves in plates showing mode conversion
}

\author{
Arno Römmeler ${ }^{\mathrm{a}, \mathrm{b}, *}$, Peter Zolliker ${ }^{\mathrm{a}}$, Jürg Neuenschwander ${ }^{\mathrm{a}}$, Valentin van Gemmeren ${ }^{\mathrm{b}}$, Mario Weder $^{\mathrm{b}}$, \\ Jürg Dual ${ }^{\mathrm{b}}$ \\ ${ }^{a}$ Empa, Swiss Federal Laboratories for Materials Science and Technology, Dübendorf, Switzerland \\ ${ }^{b}$ Institute for Mechanical Systems, ETH Zürich, 8092 Zürich, Switzerland
}

\begin{abstract}
In this paper we demonstrate a non-destructive, non-contact detection method for small defects in thin polymer plates using an air coupled ultrasonic (ACUS) setup. There exist many applications for such methods, e.g. quality control in the manufacturing process or failure prevention by periodical inspections during the lifetime of a product. We demonstrate a setup for the inspection of plates together with signal analysis algorithms to process the measured data, meeting the challenges to handle the dispersive signals and establishing a robust failure criterion.

Pressure waves from the transmitter excite different modes of Lamb waves inside the plate. These Lamb waves propagate in the plate and reradiate pressure waves into the air that are then detected by the receiver. Lamb mode conversion is used for defect detection. A numerical model allows the visualization of the propagating waves in the air as well as the Lamb waves inside the plate.

Four key parameters of the setup are identified, two angles and two distances. The transmitter and the receiver angles are used to select which Lamb mode (anti-symmetric $\mathrm{A}_{0}$ or symmetric $\mathrm{S}_{0}$ ) is mainly excited and detected, respectively. For the acquisition of the Lamb wave signal the distance from the transmitter to the receiver should be as large as possible but is limited by the attenuation of the signal. Measurements for different values of this distance are essential for signal analysis. The distance between transducer and plate surface should be as small as possible even if it may introduce secondary Lamb waves due to reflections of the pressure wave between transmitter and plate surface. Two algorithms, a model based one and a data driven one, are presented to separate Lamb modes that overlap in time. In these separated signals, the Lamb mode conversion from $\mathrm{A}_{0}$ to $\mathrm{S}_{0}$ is shown, allowing a localization of the defect.

We conclude that defect detection and localization with Lamb mode conversion is possible with an air coupled ultrasonic setup. Multiple measurements along the propagation direction of the Lamb waves are necessary to allow a thorough signal analysis and visualize the mode conversion.
\end{abstract}

Keywords: air-coupled ultrasound, non-contact, guided waves, Lamb waves, mode conversion, mode separation

\section{Introduction}

The aim of this project is to detect defects in thin polymer plates using Lamb waves in a contactless manner. The general motivation for this research is 5 industrial applications for which a single sided and contact free inspection is required. One example is

\footnotetext{
* Corresponding author

Email address: arno.roemmeler@empa.ch (Arno Römmeler)
}

inline inspection of the pipe extrusion process. In this paper, the key parameters of an air coupled ultrasonic inspection setup with Lamb waves are identified. Also two methods to analyze the measured data are presented. The detection and localization of a defect in the plate with Lamb mode conversion is shown.

Ultrasonic inspection is a non-destructive testing 15 method with a wide field of different applications. Traditionally, liquid coupled ultrasonic inspection is used together with bulk waves that propagate in- 
side the specimen. With the improvement of the air coupled ultrasonic transducers, e.g. by Hutchins et 20 al [1] or Bhardwaj [2], air coupled ultrasonic inspection became feasible.

Different air coupled inspection methods with bulk waves exist. One of them is the surface inspection by reflection as shown by Neuenschwander et al 3 .

25 For the detection of flaws inside a specimen, bulk waves can also be used in an air coupled ultrasonic inspection setup as demonstrated by Sanabria et al 4].

\subsection{Lamb waves}

The use of bulk waves requires relatively thick specimens because the signals generated by air coupled transducers are typically in the sub Megahertz regime. For thinner materials, Lamb wave inspection is better suited, as demonstrated

35 with a single sided setup by Castaigns and Cawley [5] or in a two sided setup by Hutchins et al [1 because the waves propagate along the structure and interact with the defects regardless of their position in the thickness dimension of the plate.

40 Ultrasonic air coupled inspection with Lamb waves in transmission is often used for the detection of defects in composites, especially delaminations, e.g. by Ramadas et al [6]. Ostachowicz et al [7] compare the excitation of Lamb waves with dif45 ferent types of air coupled ultrasonic transmitters for delamination detection. Kazys et al [8] show that also $\mathrm{A}_{0}$ Lamb waves can be excited with air coupled transducers that feature a group velocity that is lower than the speed of sound in the air.

50 A special case is the defect detection by Lamb mode conversion. As shown by $\mathrm{Xu}$ et al [9] (although not air coupled but liquid coupled) Lamb waves can be converted from one mode to another at the defect and thus used for defect sensing.

55 Benmeddour et al [10, 11] experimentally and numerically show that the incident $\mathrm{A}_{0}$ Lamb mode is partially converted to a $\mathrm{S}_{0}$ Lamb mode and vice ${ }_{105}$ versa at defects like steps or asymmetrical notches.

\subsection{Signal analysis}

Signal analysis is a key component of any air coupled ultrasonic inspection. Due to the fact that, depending on frequency and specimen thickness, two or more Lamb modes propagate in the 65 specimen, it is possible that these modes overlap in time and interfere.
Hayashi and Kawashima 12 present a method to separate $A_{0}$ and $S_{0}$ modes by filtering in Fourier space. This method requires a proper selection of ${ }_{70}$ the filtering parameters and separates the signal into its different modes. No additional information like the corresponding wavenumbers is gained. $\mathrm{Xu}$ et al [13] introduce a method called "dispersion compensation" to compress the Lamb waves into temporal pulses that can then be separated with rectangular windows. Cesnik and Raghavan [14] propose a way to identify and separate Lamb modes using chirplet matching and mode correlation. Vollmann et al [15] present the application of 80 Prony's method for the detection of Lamb waves and the recovery of the wavenumber and individual Lamb modes from measurements. We implement this method for our measurements because it fits our requirements and we show the performance for data from an air coupled inspection setup using Lamb waves.

\subsection{Problem description}

In our setup we have to deal with additional challenges. Comparing to Castaings and Cawley [5], who use steel specimens, the polymer plates feature a higher attenuation of the acoustic waves which restricts the maximum distance between transmitter and receiver and a much lower group velocity of the Lamb waves. Our setup is similar to the one that $\mathrm{Xu}$ et al 9] present, but the air coupled transducers are operated in a lower frequency range, resulting in pulses that are longer in their duration and propagate slower than the ones in a liquid coupled setup with steel plates. We adapt our setup to allow multiple measurements of the Lamb waves along the propagation direction and present two algorithms that are able to separate the signals into their individual modes even if they are overlapping temporally. With these adaptations we are able to show the conversion of an $\mathrm{A}_{0}$ mode to a $\mathrm{S}_{0}$ mode at the defect position.

\section{Methods}

The analytical description of Lamb waves in plates was published by Horace Lamb [16] more than 100 years ago. According to Rose [17] there 
exist two governing equations, the dispersion relation for anti-symmetric Lamb modes

$$
\frac{\tan (q h)}{\tan (p h)}=-\frac{\left(q^{2}-k^{2}\right)^{2}}{4 k^{2} p q}
$$

and the one for symmetric modes

$$
\begin{gathered}
\frac{\tan (q h)}{\tan (p h)}=-\frac{4 k^{2} p q}{\left(q^{2}-k^{2}\right)^{2}}, \\
p^{2}=\frac{\omega^{2}}{c_{1}^{2}}-k^{2}, q^{2}=\frac{\omega^{2}}{c_{2}^{2}}-k^{2},
\end{gathered}
$$

where $2 h$ is the plate thickness, $c_{1}$ and $c_{2}$ are the wave speeds of $\mathrm{P}$ - and $\mathrm{S}$-waves in the bulk, $k$ is the wavenumber and $\omega$ is the angular frequency.

In this chapter we present two methods to separate the individual Lamb modes contained in the captured signals. The first method, a model based approach, makes use of the physical description of Lamb waves in a plate to split the signal similar to the "dispersion compensation method" by Xu et al 13. The second one is data driven and known as Prony's method. It allows the separation of modes with different group velocities.

The target is to calculate the shape of the individual Lamb modes at a reference position, e.g. the position of the first measurement. All quantities denoted with a hat are in Fourier space and dependent on the frequency $\omega$, all other variables are functions of time. In Fourier space plane Lamb modes can be propagated to any other position by a multiplication with the exponential function and exponent $-i k_{m}(\omega) x$, given that the wavenumber $k_{m}$ of mode $m$ and distance $x$ are known:

$$
\hat{u}_{m}(x)=\hat{u}_{m}(0) e^{-i k_{m} x} .
$$

The measurements $\hat{b}(x)$ contain the sum of all modes at position $x$ which can then be related to the initial position:

$$
\hat{b}(x)=\sum_{m} \hat{u}_{m}(x)=\sum_{m} \hat{u}_{m}(0) e^{-i k_{m} x} .
$$

An algorithm that successfully decomposes the measured signals into individual Lamb modes therefore also generates the corresponding disper- 130 sion relation $k_{m}(\omega)$ for each Lamb mode inherently. The system is highly nonlinear because of the unknown wavenumbers $k_{m}$ in the exponential functions.

\subsection{Model based identification of Lamb modes}

For every frequency $\omega$, this problem has the following properties:

- $N$ measurements $\hat{\boldsymbol{b}}=\left[\hat{b}\left(x_{0}\right), \hat{b}\left(x_{1}\right), \cdots, \hat{b}\left(x_{N-1}\right)\right]^{\top}$ starting from the initial position $x_{0}=0$,

- $M$ Lamb modes $\hat{\boldsymbol{u}}=\left[\hat{u}_{1}, \hat{u}_{2}, \cdots, \hat{u}_{M}\right]^{\top}$ at the initial position $x_{0}=0$,

- $M$ wavenumbers $\boldsymbol{k}=\left[k_{1}, k_{2}, \cdots, k_{M}\right]^{\top}$.

The first method to solve the nonlinear problem is with the help of a solution to the Lamb wave equations. Given a set of material parameters for the plate (Young's modulus $E$, mass density $\rho$, Poisson's ratio $\nu$, thickness $2 h$ ) chosen by an educated guess, e.g. from the material data sheet, the solutions of these equations yield a dispersion relation $k_{m}(\omega)$ for every Lamb mode.

When $k_{m}$ is given, the initial problem resorts to a linear system of equations. All exponential terms in Equation (5) can then be grouped in a (known) matrix $\hat{\mathbf{P}}$ with elements

$$
\hat{P}_{n m}=e^{-i k_{m} x_{n}} .
$$

The system of equations for every frequency $\omega$ reads

$$
\hat{\mathbf{P}} \cdot \hat{\boldsymbol{u}}=\hat{\boldsymbol{b}}+\hat{\boldsymbol{e}}
$$

where $\hat{\boldsymbol{b}}$ are the Fourier transformed measurements and $\hat{\boldsymbol{u}}$ are the unknown Lamb modes at the initial position $x_{0}$ and $\hat{\boldsymbol{e}}$ contains the estimation error. If the number of measurements $N$ is larger than the number of modes $M$, the System (7) can be solved for $\hat{\boldsymbol{u}}$ by means of least squares, i.e. minimizing $\hat{\boldsymbol{e}}^{\top} \hat{\boldsymbol{e}}$. Based on $\hat{\boldsymbol{u}}$ one can also get the calculated measurements $\hat{\boldsymbol{b}}_{\text {calc }}=\hat{\mathbf{P}} \cdot \hat{\boldsymbol{u}}$.

In the time domain, the error $\boldsymbol{e}$ for the chosen set of material parameters at every position $x$ is the difference to the measured values:

$$
\boldsymbol{e}(x)=\boldsymbol{b}_{\text {calc }}(x)-\boldsymbol{b}(x) .
$$

The total error at all positions can then be minimized by a nonlinear optimizer, e.g. lsqnonlin in MATLAB to find a final set of material parameters $(E, \rho, \nu, h)$, the final dispersion relations and the amplitudes $\boldsymbol{u}$ of the separated Lamb modes at the initial position.

For our type of setup, the first three Lamb modes with their corresponding dispersion relations are sufficient and therefore we set $M=3$. 


\subsection{Data driven identification of Lamb modes}

For this method it is important to note that the measurement points have to be equidistant and therefore $x_{0}, x_{1}, \ldots x_{n} \ldots x_{N-1}$ can be rewritten as $x_{n}=n \cdot \Delta x, n=0,1, \ldots, N-1$ and $\Delta x$ has to be smaller than half of the smallest wavelength.

The basic idea is that the frequency spectrum $\hat{b}(n \cdot \Delta x)$ at the location $n$ for a frequency $\omega$ can be approximated as a polynomial

$$
\hat{b}(n \cdot \Delta x) \approx C_{1} \mu_{1}^{n}+C_{2} \mu_{2}^{n}+\ldots+C_{Q} \mu_{Q}^{n},
$$

where $Q$ is the polynomial order and $\mu_{i}=e^{\kappa_{i} \Delta x}$ where $\kappa_{i}$ is a complex number. This leads to a system of equations:

$$
\begin{array}{rlrl}
\hat{b}\left(x_{0}\right) & =C_{1} \quad+C_{2} \quad+\ldots+C_{Q} \\
\hat{b}\left(x_{1}\right) & =C_{1} \mu_{1} \quad+C_{2} \mu_{2} \quad+\ldots+C_{Q} \mu_{Q} \\
\hat{b}\left(x_{2}\right) & =C_{1} \mu_{1}^{2}+C_{2} \mu_{2}^{2} \quad+\ldots+C_{Q} \mu_{Q}^{2} \\
\ldots & =\ldots & \\
\hat{b}\left(x_{N-1}\right) & =C_{1} \mu_{1}^{N-1}+C_{2} \mu_{2}^{N-1}+\ldots+C_{Q} \mu_{Q}^{N-1} .
\end{array}
$$

Note that the coefficients $C$ are equivalent to the elements of $\hat{\boldsymbol{u}}$ in Section 2.1. We introduce a polynomial with the roots $\mu_{1}, \mu_{2}, \ldots \mu_{Q}$ and coefficients $\alpha_{1}, \alpha_{2}, \ldots \alpha_{Q}$ :

$$
\mu^{Q}+\alpha_{1} \mu^{Q-1}+\ldots+\alpha_{Q-1} \mu+\alpha_{Q}=0 .
$$

With this polynomial we can apply a transformation to Equation (10) as described by Vollmann et al 15 and obtain a system that is linearly dependent on $\boldsymbol{\alpha}$ instead of the exponential terms $\boldsymbol{\mu}$.

$$
\begin{gathered}
\mathbf{A} \boldsymbol{\alpha}=\mathbf{d} \\
\mathbf{d}=\left[\begin{array}{c}
-\hat{b}(Q) \\
-\hat{b}(Q+1) \\
\ldots \\
-\hat{b}(N-1)
\end{array}\right] \\
\mathbf{A}=\left[\begin{array}{cccc}
\hat{b}(Q-1) & \hat{b}(Q-2) & \ldots & \hat{b}(0) \\
\hat{b}(Q) & \hat{b}(Q-1) & \ldots & \hat{b}(1) \\
\ldots & & & \\
\hat{b}(N-2) & \hat{b}(N-3) & \ldots & \hat{b}(N-Q-1)
\end{array}\right]
\end{gathered}
$$

To find a solution to this system of equations, the singular value decomposition turns out to be helpful. We introduce the augmented matrix $[\boldsymbol{d}, \mathbf{A}]$ and calculate the decomposition

$$
[\mathbf{U}, \mathbf{S}, \mathbf{V}]=\operatorname{svd}([\boldsymbol{d}, \mathbf{A}]),
$$

where $\mathbf{U}$ and $\mathbf{V}$ are matrices containing the left and right singular vectors respectively and $\mathbf{S}$ is a diagonal matrix containing the singular values $s_{i}$.

For the system of equations 12 we can find solutions $\boldsymbol{\alpha}$ as shown by Tirendi and Martin 18:

$$
\boldsymbol{\alpha}=-\frac{\sum_{j=m_{e}+1}^{Q+1} \mathbf{V}(1, j)^{*} \cdot \mathbf{V}(1,2: e n d)}{\sum_{j=m_{e}+1}^{Q+1}|\mathbf{V}(1, j)|^{2}},
$$

where $m_{e}$ is the number of expected modes. For our measured signals, we use $m_{e}=2$ and $Q=16$. In our case the measurements also contain a third propagating Lamb mode, $\mathrm{A}_{1}$. But choosing $m_{e}=3$ or higher does not help to separate the $S_{0}$ and $A_{1}$ modes.

From the singular value decomposition we know that the singular values and singular vectors are ordered by magnitude of the singular value. The set of solutions $\boldsymbol{\alpha}$ are the coefficients of the polynomial in Equation (11). To find the wavenumbers of the propagating modes, we need to solve the polynomial in Equation (11) for $\boldsymbol{\mu}$ and then derive the coefficients $C$ from Equation $(10)$. Finally, we can calculate $\boldsymbol{\kappa}$ :

$$
\boldsymbol{\kappa}=\ln (\boldsymbol{\mu}) / \Delta x .
$$

and the wavenumbers $\boldsymbol{k}$ of the propagating modes are the imaginary parts of $\boldsymbol{-} \boldsymbol{\kappa}$. The real part of $\kappa$ is related to the measured attenuation, which is a combination of attenuation of the Lamb waves, the loss because of the reradiation into the air and beam widening.

Since in general this polynomial is of higher degree than four, no analytical solution is available and one needs to calculate the roots numerically. The other words, $\boldsymbol{\mu}$ is not ordered in the same way as $\boldsymbol{\alpha}$ or $\mathbf{S}$ but by magnitude of $\boldsymbol{\mu}$. As a result, this method does not yield dispersion relations $k(\omega)$, but a set of wavenumbers $k$ and the corresponding

150 frequencies $\omega$. However, for easy cases the dispersion curves can be connected manually as shown in Section 6.2 because the dispersion relation in general changes smoothly with frequency.

\section{Setup and Measurements}

In total, three different experiments where carried out. The first one aims to characterize the general setup. Therefore the complete signal including 
the air signal is captured with a large amplitude range. The second one is used to develop the signal nalysis algorithms, focusing on the Lamb wave sig- 210 nal that arrives first. The amplitude range is small to reflect the small amplitudes of the Lamb wave signal and the air signal is only partially recorded. Both experiments are carried out with a flawless plate specimen. The third one uses a plate with an artificial defect and is used to demonstrate defect detection by Lamb mode conversion.

Figure 1 shows the basic setup with the four key parameters, the transmitter angle $\alpha_{T x}$, the receiver angle $\alpha_{R x}$, the transmitter to receiver distance ${ }_{220}$ $d_{T x R x}$ and the transducer to specimen surface distance $d_{S}$ (transmitter and receiver are mounted at the same distance to the specimen surface). For the rotation of the transmitter $\left(\alpha_{T x}\right)$ and receiver $\left(\alpha_{R x}\right)$ we use two manual goniometers. For prac- ${ }_{225}$ tical reasons, the center of rotation lies inside the transducers, $10 \mathrm{~mm}$ away from the active surface on the dashed-dotted line shown in Figure1. One stepper motor controller and driver is used to control the distance $d_{T x R x}$ by moving the receiver and an- ${ }_{230}$ other one to change $d_{S}$ by lifting both transducers.

The electrical equipment for our measurements (in 235 order of the signal chain) consists of a signal gener185 ator (NI PCI-5421, National Instruments, Austin, TX, USA), a signal amplifier (Ritec RPR-4000, RITEC Inc., Warwick, RI, USA) for driving the transmitter, a pair of air coupled ultrasonic transducers (Ultran NCG200D25 \& Ultran NCG200D13, The Ultran Group Inc., State College, PA, USA), a preamplifier (Panametrics), the Ritec amplifier again, a bandpass filter (Krohn Hite 3944) and an AD converter (NI PCI-5122).

The electrical excitation of the transmitter is a pulse of 1.5 periods of a sine function with $181 \mathrm{kHz}$, windowed by a Gaussian window. The signal amplitude is up to $500 \mathrm{~V}$. Averaging is used with up to 1024 samples per measurement point. Contin- ${ }^{250}$ uous (harmonic) excitation is not feasible due to the direct signal through the air that masks the Lamb wave signal. The received signal is sampled at $10 \mathrm{MHz}$ (NI PCI-5122). For excitation, the transducer with a larger diameter $(25 \mathrm{~mm})$ is used, the diameter of the receiver is $12.5 \mathrm{~mm}$. The benefit of ${ }_{255}$ the larger diameter transducer is a stronger Lamb wave, while the one with a smaller diameter at the receiver yields better localization of the signal.

\subsection{Numerical model}

A two-dimensional numerical model is set up in COMSOL to study effects that are difficult to visualize experimentally, e.g. the pressure field between transmitter and plate and the displacement field in the plate. Also the dependency of the excited Lamb modes on the transmitter angle is studied numerically. The two dimensional transient model is composed out of two domains, the air and the plate. The plate is modeled as solid with a height of $3 \mathrm{~mm}$ and a length of $240 \mathrm{~mm}$. It features a linear elastic isotropic material model with Young's modulus $E=5.2 \mathrm{GPa}$, density $\rho=1190 \mathrm{~kg} / \mathrm{m}^{3}$ and Poisson's ratio $\nu=0.32$ corresponding to the properties of PMMA. Plane strain is assumed. The mesh for the plate uses brick elements with 16 elements in thickness direction of the plate. At the short ends of the plate, low reflective boundary conditions are applied to prevent reflections.

The air is modeled as gas with a density of $1.2 \mathrm{~kg} / \mathrm{m}^{3}$ and a speed of sound of $343 \mathrm{~m} / \mathrm{s}$. The domain has a size of $240 \mathrm{~mm}$ by $35 \mathrm{~mm}$ and is surrounded by perfectly matched layers to prevent reflections of the pressure waves that leave the computational domain. The mesh for the air consists of triangular elements with a maximum length of $0.5 \mathrm{~mm}$ to allow an adaption of the mesh to the transmitter and receiver angle. The transducers are modeled as rigid boxes inside the air.

The maximum time step for the iterative solver is $0.1 \mu \mathrm{s}$. In contrast to the excitation with the Ultran transducers that have an inhomogeneous pressure distribution in the near field, the excitation in the numerical model is created by a prescribed pressure on the surface of the transmitter with three periods of a sine wave $(f=181 \mathrm{kHz})$ windowed with a Gaussian window in time and space. For the experiment, 4 an electrical pulse with only 1.5 periods of a sine wave is used for the excitation of the transducer, resulting in a pressure wave that is longer than 1.5 periods. In the simulation, the effects of the piezoelectric transducer is not modeled, but pressure wave is 3 periods long.

\subsection{Specimen}

For this study, $3 \mathrm{~mm}$ thick polymer plates are used as specimen. PMMA is chosen as material because of its low acoustic damping. To minimize the chance of recording Lamb waves that are reflected at the object boundaries, a sufficiently large plate is chosen $(400 \mathrm{~mm} \times 400 \mathrm{~mm})$. The setup is characterized with a flawless plate, for the defect detection 


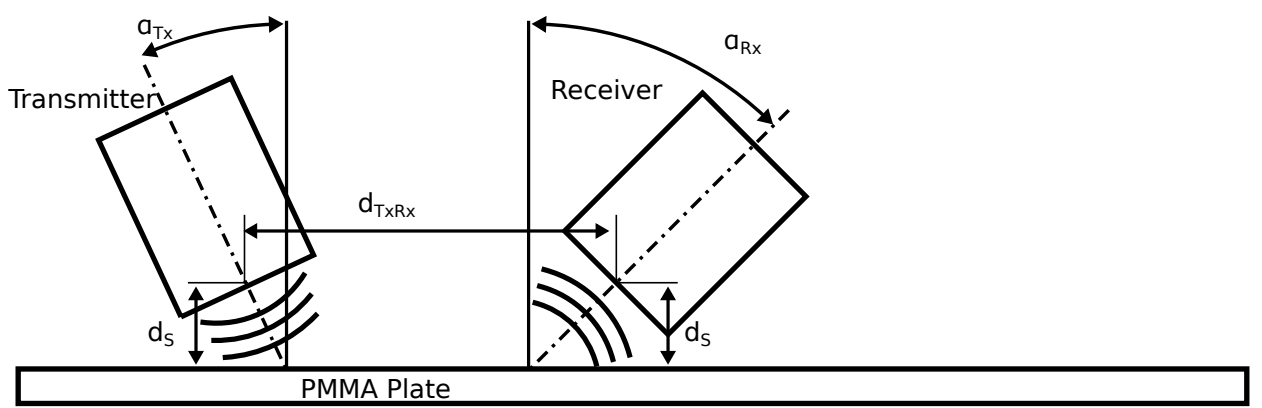

Figure 1: Basic setup with key parameters for an air coupled ultrasonic inspection.

a hole is drilled in the center of the plate with dimeter of $2 \mathrm{~mm}$ and depth of $1.5 \mathrm{~mm}$ as it can be seen in Figure 3. This defect is a three-dimensional 285 defect. Other types of defects, e.g. two-dimensional defects (cracks or delaminations) are also possible if they induce Lamb mode conversion.

\subsection{The air signal}

The air signal includes the wave that is traveling directly from the transmitter to the receiver as well as those waves that are reflected at different surfaces before reaching the receiver. It has an amplitude up to $10 \mathrm{~V}$ and lasts as long as $2000 \mu \mathrm{s}$. The effect of the distances $d_{T x R x}$ and $d_{S}$ is investigated with a flawless thin plate. A study for both parameters is carried out as a 2D-scan as shown in Figure 2 by varying $d_{S}$ from $8-68 \mathrm{~mm}$ in steps of $2 \mathrm{~mm}$ and $d_{T x R x}$ from $60-140 \mathrm{~mm}$ in steps of $0.5 \mathrm{~mm}$ for every step of $d_{S}$ as shown in Figure 2. For this experiment, the transmitter angle $\alpha_{T x}$ is $9^{\circ}$ and the receiver angle $\alpha_{R x}$ is $19^{\circ}$.

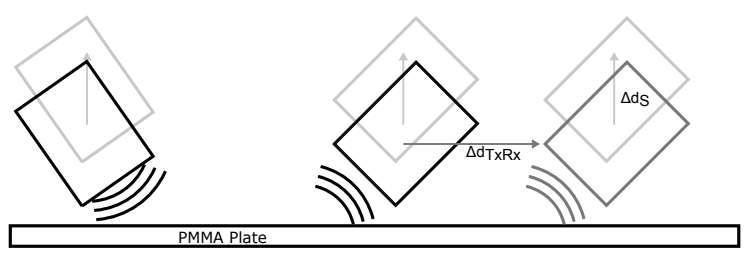

Figure 2: Setup to characterize the air coupled ultrasonic inspection method with a flawless plate. The effect of the transmitter - receiver distance $d_{T x R x}$ and the transmitter specimen surface distance $d_{S}$ is investigated.

\subsection{The Lamb wave signal}

For the development of signal analysis algorithms, the Lamb wave signal was recorded at 305 a large number of measurement points along the propagation path for a specimen without defect. 845 equispaced measurements along the propagation direction are taken with a spacing of $0.2 \mathrm{~mm}$, in total $169 \mathrm{~mm}$. The Lamb wave signal features a small amplitude up to $100 \mathrm{mV}$ and arrives before the air signal, in general within $500 \mu \mathrm{s}$. For this ex290 periment, the transmitter angle $\alpha_{T x}$ is $9^{\circ}$ and the receiver angle $\alpha_{R x}$ is $19^{\circ}$.

\subsection{Defect detection by Lamb mode conversion}

To show the defect detection by Lamb mode conversion, a thin plate with a defect is examined. 320 equispaced measurements along the propagation direction are taken with a spacing of $0.5 \mathrm{~mm}$, in total about $160 \mathrm{~mm}$ as shown in Figure 3. The defect is located $30 \mathrm{~mm}$ away from the position of the first measurement. For this experiment, the transmitter angle $\alpha_{T x}$ is $19^{\circ}$ and the receiver angle $\alpha_{R x}$ is $9^{\circ}$.

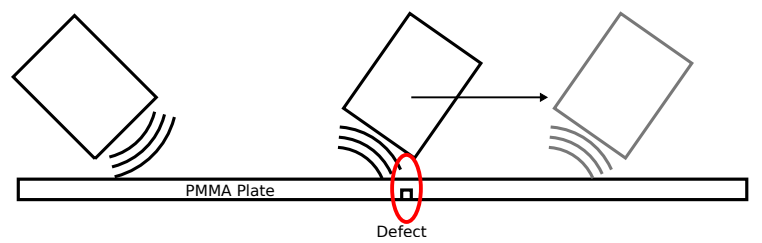

Figure 3: Setup for an air coupled ultrasonic inspection by Lamb mode conversion. The receiver is moved along the plate surface, passing the location of the defect.

\section{Characteristics of an air coupled ultra- sonic inspection setup}

In an air coupled ultrasonic inspection experiment with Lamb waves, two major signal components are contained in the receiver data. First, the 
Lamb wave signal that travels along the plate and may carry information about defects in the specimen and second the direct signal through the air. The setup is made such that the first one generally arrives first because of the higher group velocity of the Lamb waves in the plate. The second one arrives later and is more than one order of magnitude stronger. Since it does not penetrate into the plate, it carries no valuable information and can be discarded.

This section shows the components and variation of the recorded signal for a flawless plate. This signal is considered the base signal that is always present for this type of investigation and may be altered by the presence of defects in the specimen as shown in section 6.4

The goal of this section is to choose the parameters $\alpha_{T x}, \alpha_{R x}, d_{T x R x}$ and $d_{S}$ in such a way that possible changes of the signal caused by defects are in

325 the measurement range and not masked by other signals.

\subsection{Excitation}

Since the beam diameter of the ACUS transducers (Ultran NCG200D25) is at least some multiples of the wavelength, the source of the Lamb waves at the plate surface can not be considered as a point source. Instead, a plane wave is assumed to be excited with the direction of propagation given by the transmitter.

\subsection{Effect of the transmitter angle $\alpha_{T x}$ and the re-} ceiver angle $\alpha_{R x}$ on the excited and detected Lamb modes

The numerical model is used to study which Lamb modes are excited inside the plate depending on the angle of the incident pressure wave. The out- 370 of-plane displacement of the plate surface is split into the $\mathrm{A}_{0}$ and $\mathrm{S}_{0}$ Lamb modes and shown as a function of the transmitter angle $\alpha_{T x}$ in Figure 4 For a transmitter frequency of $181 \mathrm{kHz}$ and a $3 \mathrm{~mm}$ thick PMMA plate, the optimal excitation angle is ${ }_{375}$ $19^{\circ}$ for the $\mathrm{A}_{0}$ Lamb mode and $9^{\circ}$ for the $\mathrm{S}_{0}$ Lamb mode. The $\mathrm{A}_{0}$ mode can be excited in an almost pure fashion, whereas the $\mathrm{S}_{0}$ one is excited together with an $\mathrm{A}_{0}$ Lamb mode of lower amplitude. Similar effect occur at the receiver. The receiver angle can 380 only be set to match the angle to detect one specific Lamb mode, all other Lamb modes will be captured with a lower amplitude. Details can be found in a previous study [19.

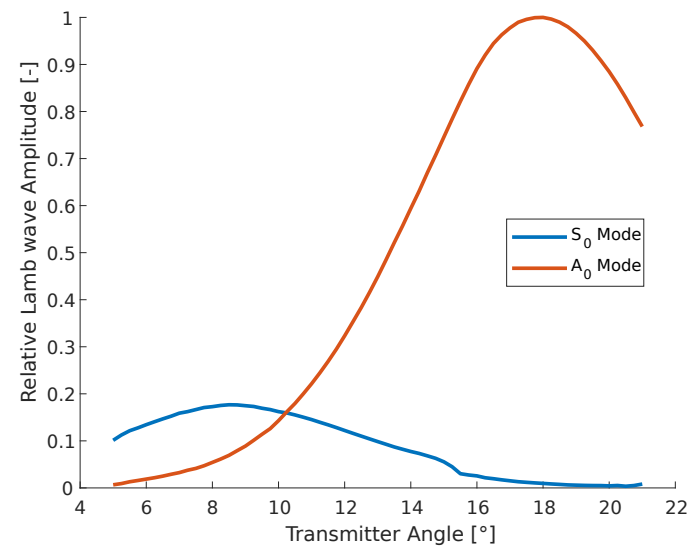

Figure 4: Results of the numerical study on the effect of the transmitter angle on the excited Lamb modes in a $3 \mathrm{~mm}$ PMMA plate and a frequency of $181 \mathrm{kHz}$. The optimal angle for the excitation of the $A_{0}$ mode is $19^{\circ}$ and the one for the $\mathrm{S}_{0}$ mode is $9^{\circ}$.

For the specific case of Lamb mode conversion, the transmitter angle has to be $9^{\circ}$ and the receiver angle $19^{\circ}\left(\mathrm{S}_{0}\right.$ to $\left.\mathrm{A}_{0}\right)$ or vice versa to detect a different mode than the one that is excited.

\subsection{The air signal: effect of $d_{S}, d_{T x R x}$}

This paragraph describes the main propagation paths of the excited pulse in the air, which is the one directly from the transmitter towards the receiver and those which include one or more reflections at specimen or equipment surfaces. The direct air signal causes two effects. First, the time of arrival of this signal represents the end of the detection window for Lamb waves, because it later masks the Lamb wave signal due to its high amplitude. As a second effect, multiple reflections of the pressure wave between transmitter and specimen surface also create multiple Lamb wave pulses inside the plate, one at each impact at the plate surface. These Lamb wave pulses originate from different points on the plate surface and, because of the different angles at each reflection, also contain a different combination of Lamb modes.

The direct air signal propagates with the speed of sound in air, which is significantly lower than the Lamb mode group velocity in polymer plates at the considered frequency. An increasing distance between transmitter and receiver $\left(d_{T x R x}\right)$ therefore is also increasing the time window to capture Lamb waves. On the other hand, with an increasing $d_{T x R x}$ the Lamb wave signal strength decreases 
because of attenuation and the loss from the reradiation into air. A good compromise has to be found ${ }_{415}$ for $d_{T x R x}$ that allows enough time to capture all relevant Lamb waves and also conserves enough signal strength. When the transducer to specimen distance $d_{S}$ is increased, the arrival time of the direct air signal ${ }_{420}$ stays constant, but the Lamb wave signal gets delayed. For this reason, it is important to keep the distance $d_{S}$ small. For geometric reasons, a low distance $d_{S}$ increases the number of reflections of the excited pulse between transmitter and plate surface. Additionally, the time between these reflections is also shorter. A low distance $d_{S}$ therefore increases the number of Lamb wave pulses created by "secondary" impacts and reduces the time in between these pulses. Finally, with a low distance $d_{S}$ the plate surface is in the near field range of the transducer.

Figure 5 shows possible paths of the air signal. The

solid line represents the direct way from transmitter to the receiver. The dashed line shows the signal that is reflected once at the plate surface in the middle between transmitter and receiver. The dotted line represents a wave pulse that undergoes one additional reflection between plate surface and transmitter surface (creating Lamb waves at the "secondary" impact as shown in blue).

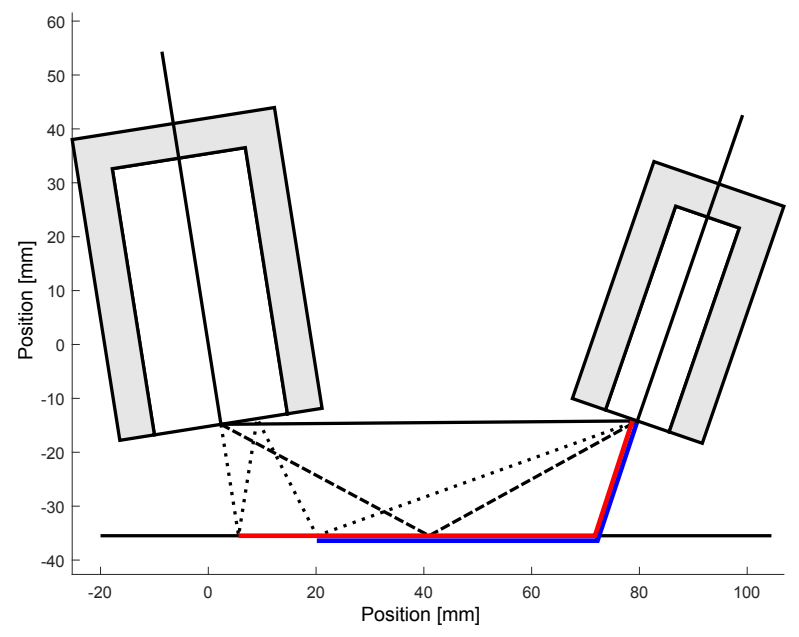

Figure 5: Some possible paths (centerlines) for the direct air signal from the transmitter (left) to the receiver (right): direct (solid), one (dashed) or two (dotted) reflections of the signal at different interfaces of the specimen or the equipment. Primary Lamb waves in the plate are shown in red, secondary in blue.

In Figure 6 a) the effect of an increased distance
$d_{T x R x}$ is shown for $d_{S}=60.5 \mathrm{~mm}$. As expected, the delay of the direct signal increases linearly with $d_{T x R x}$, whereas the delay of the signals with one (dashed) or two (dotted) reflections follow the nonlinear behavior computed according to the propagation paths shown in Figure 5. One can also see more signals that arrive even later. These originate from signals that undergo even more reflections.

Figure 6 b) shows the wave packets for a constant distance $d_{T x R x}$ and an increasing distance $d_{S}$. As expected, the arrival time of the direct signal (solid) is constant because $d_{T x R x}$ does not change. The pulses with one or more reflections overlap for small values of $d_{S}$ and separate for larger values.

a)
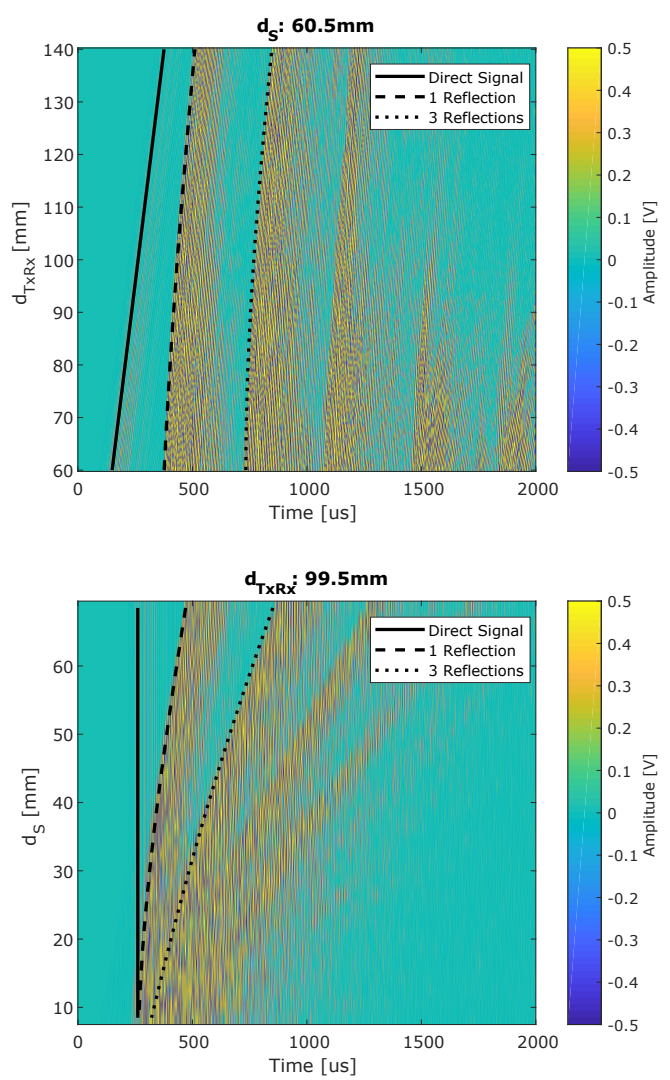

Figure 6: The variation of the air signal is shown for an increasing distance $d_{T x R x}$ in a) and $d_{S}$ in b). The signal is limited to $\pm 0.5 \mathrm{~V}$. The time of the first arrival of the direct signal (solid) and the signals with one (dashed) or two (dotted) reflections are calculated with a speed of sound in air $(343 \mathrm{~m} / \mathrm{s})$ and the propagating paths as shown in Figure 5

Figure 5 shows the path for a pressure wave that is reflected once between transmitter and plate surface (dashed line). The pressure field for this specific wave can easily be visualized with the help 
of a simulation, something that is very difficult to achieve experimentally. In Figure 7, the calculated pressure field as well as the out-of-plane inside the plate are plotted for several time steps. The pressure wave travels towards the plate $(\mathrm{a}, \mathrm{b})$ and is reflected back to the transmitter (c, d, e) while also generating the primary Lamb waves. When the wave reaches the transmitter sur-

440 face again, it is reflected a second time (f, g, h). Then the wave reaches the plate $(i, j)$ with a larger incident angle than for the first time. Therefore secondary Lamb waves are created with a different composition of Lamb modes than in the first time.

One can conclude that the direct air signal determines two important parameters, $d_{S}$ and $d_{T x R x}$. As one can see in Figure 6 , the first arrival of the direct air signal is around 150-300 $\mathrm{s}$, marking the end of the Lamb wave capture range, and extends up to $2000 \mu \mathrm{s}$. Multiple reflections complicate the situation by generating more Lamb wave pulses with the "secondary" impacts on the plate surface.

\subsection{The Lamb wave signal}

This paragraph describes the part of the signal hat can be captured before any air signal arrives. The Lamb waves inside the plate are excited when the pressure wave in the air hits the plate surface. Because of the high impedance mismatch between air and polymer, a great part of the energy is relected and only little is transmitted into the plate. Therefore, the magnitude of the Lamb wave signal is more than an order of magnitude weaker then the air signal (hence it is not visible in Figure 6). The Lamb waves propagating inside the plate reradiate captured with an air coupled receiver. They can be identified as Lamb waves by their group velocity. The primary impact of the excited pulse on the plate surface creates Lamb waves (marked with a red ellipse in Figure 8 traveling in the plate towards the receiver. One can identify at least two different Lamb modes by looking at their different propagation speeds in Figure 8 . The first one can be attributed to the $\mathrm{S}_{0}$ Lamb mode (fast, steep slope) while the second one to the $\mathrm{A}_{0}$ Lamb mode (slow, less steep slope). In fact, as we will see in Section 5 this pulse consists of three different modes.

The second $\mathrm{A}_{0}$ pulse (marked in blue) that is even higher in amplitude than the one in front is generated by a "secondary" impact of the excited pulse in air on the plate surface. Since the angle increases for the "secondary" impact on the plate surface,

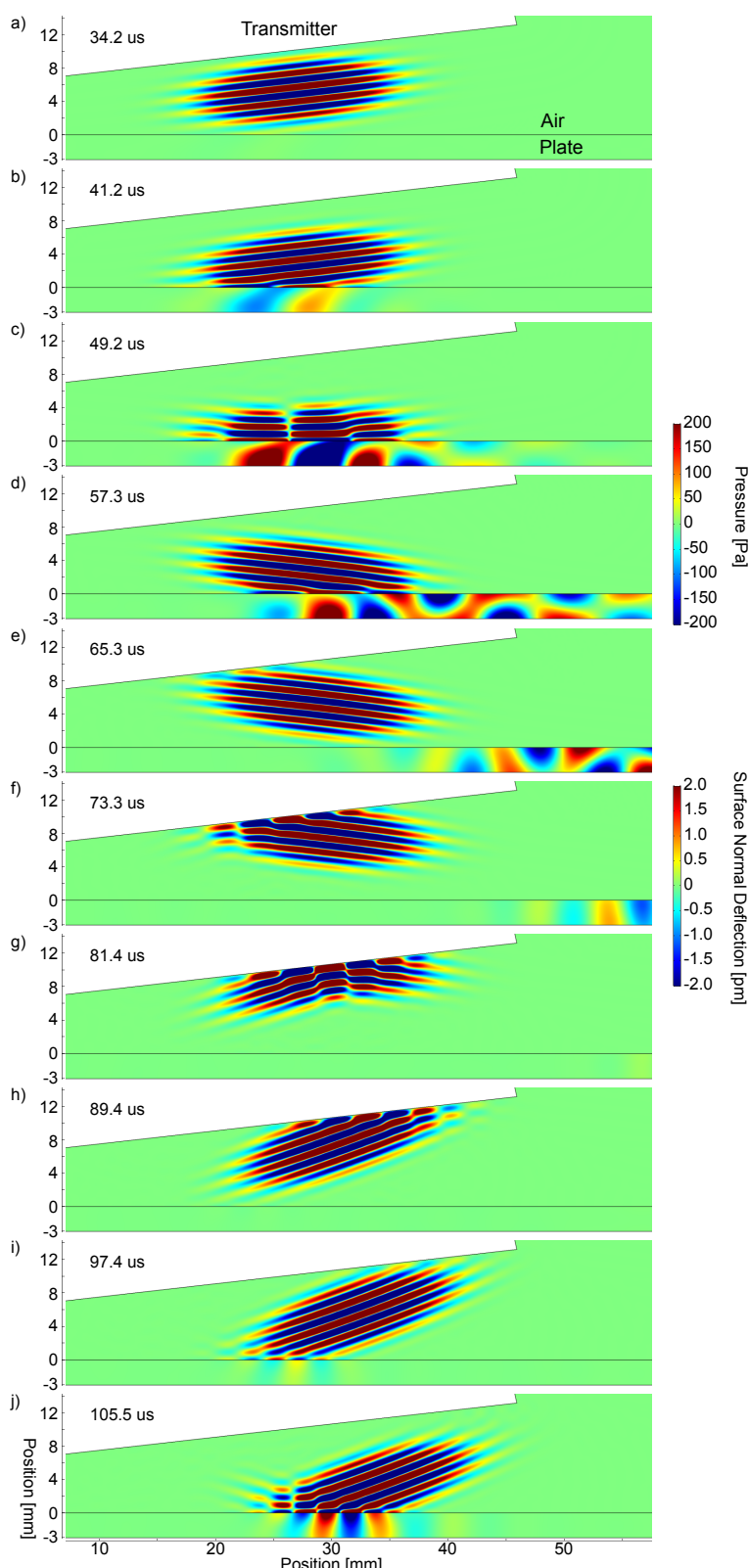

Figure 7: Simulated pressure field in the air as well as outof-plane deflection in the plate for different time steps. The pressure field is clipped to $200 \mathrm{~Pa}$, the deflection to $2 \mathrm{pm}$. The pressure wave propagates towards the plate $(\mathrm{a}, \mathrm{b})$, is reflected back and creates the primary Lamb waves in the plate $(\mathrm{c}, \mathrm{d}, \mathrm{e})$. The pressure wave is reflected again at the transmitter $(\mathrm{f}, \mathrm{g}, \mathrm{h}$ ) and reaches the plate a second time (i, $\mathrm{j}$ ), inducing secondary Lamb waves. The incident angle is higher for the second impact, leading to a different composition of Lamb modes.

the incident angle comes close to the optimal angle for the excitation of a $\mathrm{A}_{0}$ wave. This effect leads 
to the larger amplitude of the "secondary" Lamb waves. When increasing $d_{S}$ (not shown here), this pulse is delayed more than the first $\mathrm{A}_{0}$ and $\mathrm{S}_{0} \mathrm{Lamb}$ waves, confirming the assumption that this pulse is originating from a "secondary" impact.

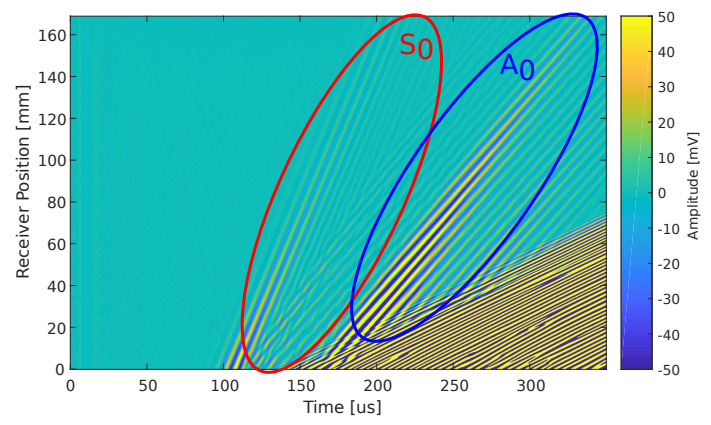

Figure 8: The measured Lamb waves that arrive before the first direct wave through air (bottom right). One can clearly identify the primary $S_{0}$ and $A_{0}$. The second $A_{0}$ wave that follows after the first one is generated by the pressure wave that is reflected between transducer and specimen surface.

\section{Signal preconditioning}

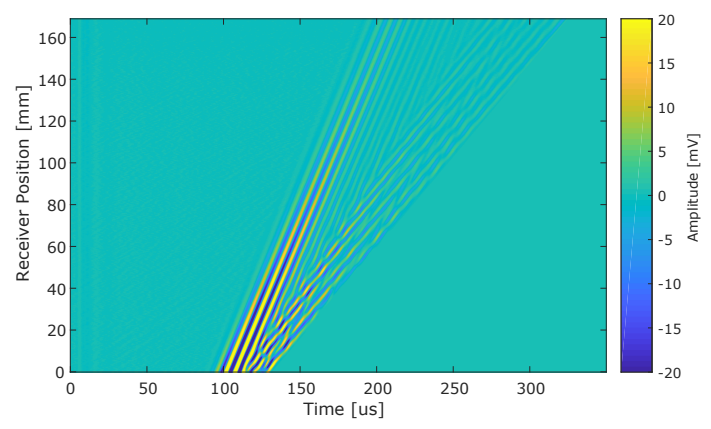

Figure 9: $S_{0}$ and $A_{0}$ Lamb waves separated from all other signals that arrive later. This data is taken to develop and 520 test the signal analysis algorithms.

As a first step, the air signal has to be removed from the data. This is done by multiplying with a Gaussian window function that removes every- 525 thing except the primary Lamb waves. Note that, because of windowing, errors are introduced in the signal. Because of the dispersion of the Lamb waves the shape of these waves changes and it is difficult to separate the pulses in the time domain. The re- 530 sult of the windowing process is shown in Figure 9 . The measurements can also be Fourier transformed. Figure 10 shows the data in wavenumber-frequency space. One can see that besides the two major modes $\left(\mathrm{A}_{0}\right.$ and $\left.\mathrm{S}_{0}\right)$, which can directly be recognized also in the untransformed data, a third mode $\left(\mathrm{A}_{1}\right)$ exists $(250-300 \mathrm{kHz})$.

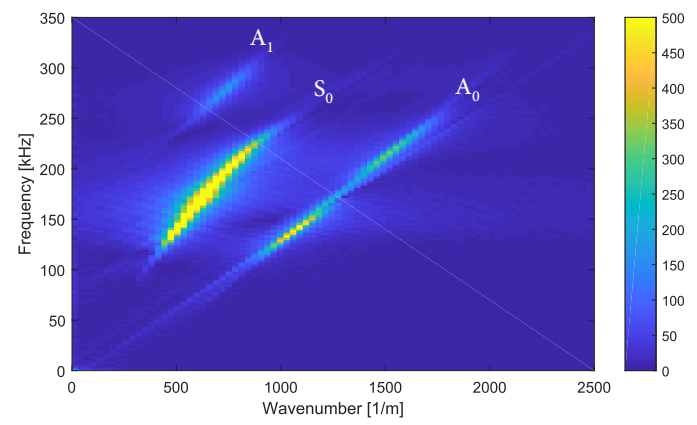

Figure 10: The measured signal (as shown in Figure 9 , Fourier transformed to the wavenumber-frequency space. One can see that besides the two major modes $\left(A_{0}, S_{0}\right)$ a third mode $\left(A_{1}\right)$ exists.

After this step of preparation, the data is analyzed with both Lamb wave separation methods presented in Section 2.

\section{Results}

This section presents the results for the Lamb mode extraction from the measured data. Both, the model based and the data driven approach yield good results in separating overlapping Lamb modes into their components and have different advan515 tages and disadvantages.

\subsection{Lamb wave model}

The Lamb wave model based method generates dispersion relations $k(\omega)$ for the specimen. These dispersion relations are shown in Figure 11 a) on top of the Fourier transformed measurement data. The $\mathrm{A}_{0}$ mode is shown in blue, the $\mathrm{S}_{0}$ mode in red and the $A_{1}$ mode in yellow.

The model covers the whole frequency range, not only the range that is shown in the graph and fit very precisely to the measured data in the region between $100 \mathrm{kHz}$ and $300 \mathrm{kHz}$.

Figure 11 b) shows the sum of the three modes in blue against the original measurement in black. There is a good agreement of the calculated result with the measurement. Notable differences occur in the range where the original function already reach zero (by windowing) and the algorithm 
a)

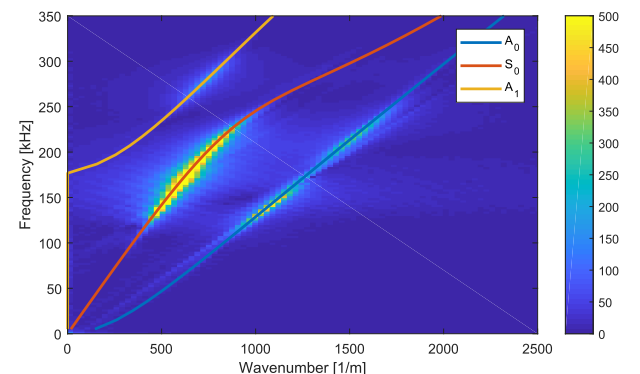

b)
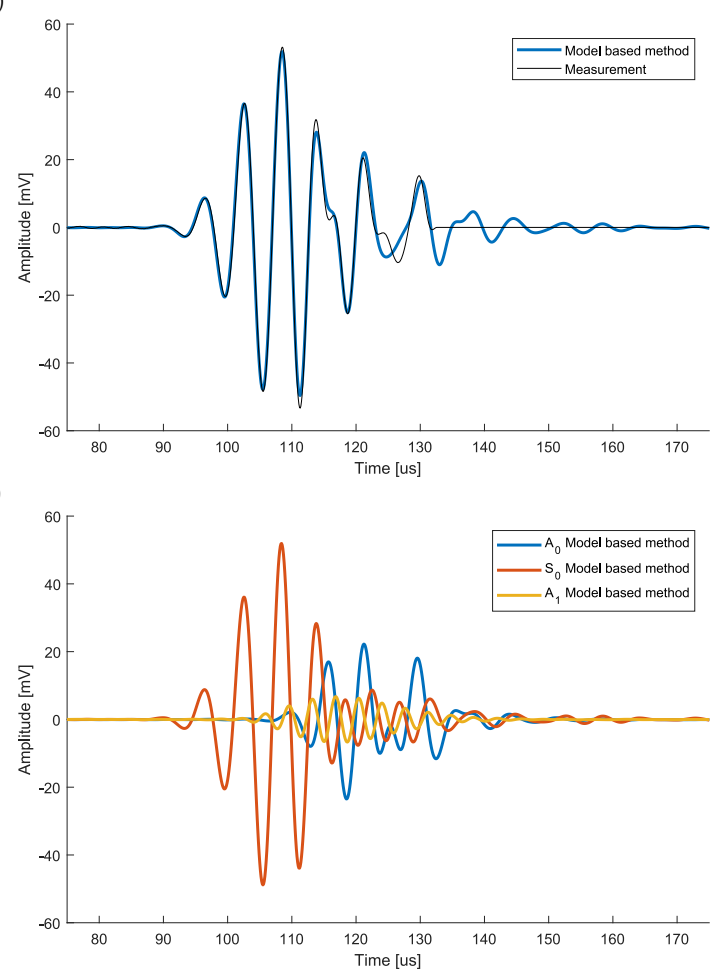

Figure 11: a) shows the calculated dispersion relation for 575 the Lamb wave model $\left(\mathrm{A}_{0}, \mathrm{~S}_{0}\right.$ and $\left.\mathrm{A}_{1}\right)$ on top of the measured data in the $k-\omega$ space. b) shows the sum of all three extracted Lamb modes and the measured data in the time domain. c) shows the individual Lamb modes in the time domain.

output does not. This is discussed in Section 7 The extracted $\mathrm{A}_{0}$ (red), $\mathrm{S}_{0}$ (blue) and $\mathrm{A}_{1}$ (yellow) Lamb modes are shown in Figure $11 \mathrm{c}$ ).

The material parameters for the PMMA plate estimated with the model are $E=3.6 \mathrm{GPa}, \rho=1171 \mathrm{~kg} / \mathrm{m}^{3}, \nu=0.34 \quad$ and $2 h=2.9 \mathrm{~mm}$. The thickness estimation corresponds to the measured thickness of the plate very well.

\subsection{Data driven method}

The result of Prony's method is a set of wavenum545 bers and their corresponding frequency, which are a priori not connected to a dispersion relation. After exclusion of the wavenumbers with high imaginary part that do not resemble propagating waves, the result is presented Figure 12 a). Visually, two 550 dispersion relations can be recognized but the single wavenumbers (red dots) are not yet connected. In this case they can be separated easily because for each wavenumber, the lower point (frequency) can be assigned to the $A_{0}$ branch and the upper 555 one to the combined $\mathrm{S}_{0}$ and $\mathrm{A}_{1}$ branch. As one could see, this method somehow mixes $S_{0}$ and $A_{1}$ Lamb modes by introducing additional points at the transition between those two modes. Again, one could split both modes manually, e.g. by assigning all wavenumbers that have a frequency below $240 \mathrm{kHz}$ to the $\mathrm{S}_{0}$ mode and the remaining ones to the $\mathrm{A}_{1}$ mode.

The recovered combined signal $\left(A_{0}, S_{0}\right.$ and $\left.A_{1}\right)$ is shown in blue together with the original signal (black) in Figure 12 b). Also for this method, the result is in good accordance with the measurements. Again, there is some deviation where the original signal has zero level.

The wavenumbers assigned to the $\mathrm{A}_{0}$ branch and mode (blue), all other wavenumbers yield the combined $\mathrm{S}_{0}$ and $\mathrm{A}_{1}$ branch (red) as plotted in Figure 12 c).

\subsection{Comparison}

The requirements on the position of the measurement points is less restrictive for the model based method, because Prony's method needs equidistant measurements. The model based approach could also be extended to other waves than plane waves by including a term for the wave amplitude that is dependent on the position of the measurement. The data driven method is orders of magnitudes faster because it does not rely on a nonlinear optimization process.

\subsection{Defect detection with Lamb mode conversion}

The measurements at each receiver position from $0 \mathrm{~mm}$ to $100 \mathrm{~mm}$ are shown as B-scan in Figure 13 a). Please note that the amplitude is limited to $\pm 5 \mathrm{mV}$ for better visualization of the weaker $\mathrm{S}_{0}$ sig590 nal. The defect position at $35 \mathrm{~mm}$ is marked by the 
a)

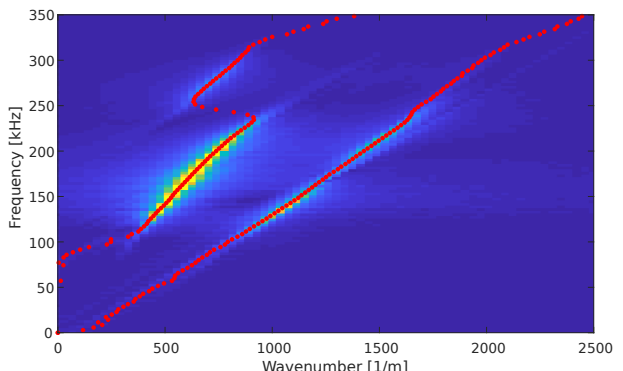

b)
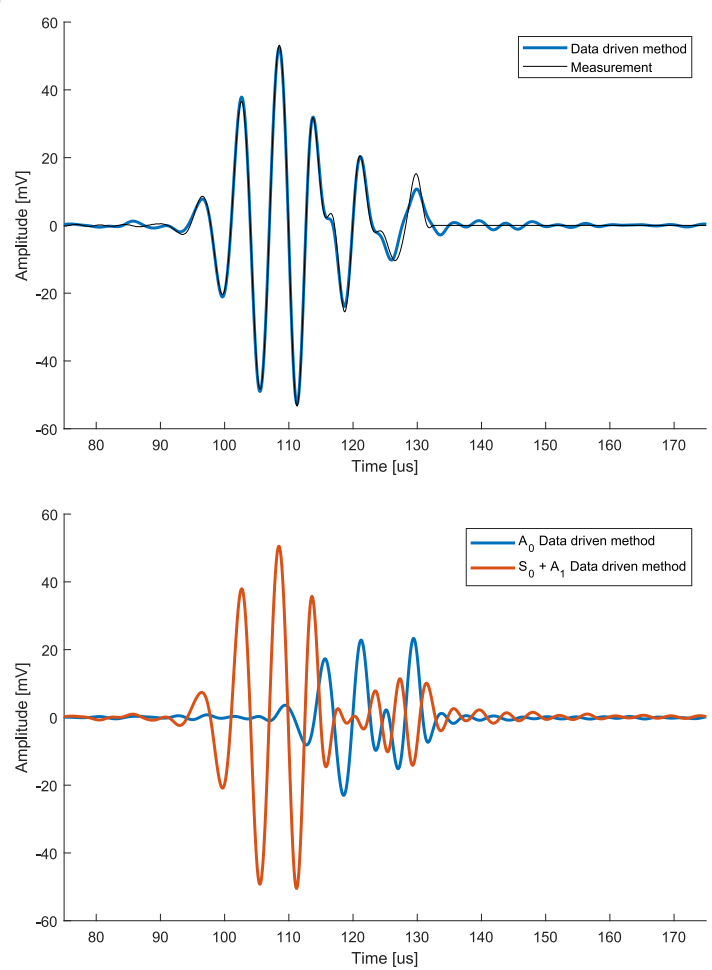

Figure 12: a) shows all the extracted wavenumbers and the corresponding frequencies as red dots on top of the measured data in the $k-\omega$ space. b) shows the sum of the extracted Lamb modes and the measured data in time domain. c) shows the separated Lamb modes $\left(\mathrm{A}_{0}, \mathrm{~S}_{0}+\mathrm{A}_{1}\right)$ in time domain.

horizontal dashed line.

The decomposition of the measurements with the data driven method yields the stronger $\mathrm{A}_{0}$ Lamb mode as shown in Figure 13 b) with an amplitude range of $\pm 50 \mathrm{mV}$ and the weaker $\mathrm{S}_{0}$ Lamb mode shown in Figure 13 c).

Because the defect is in the middle of the specimen and the initial part of the measurement does not
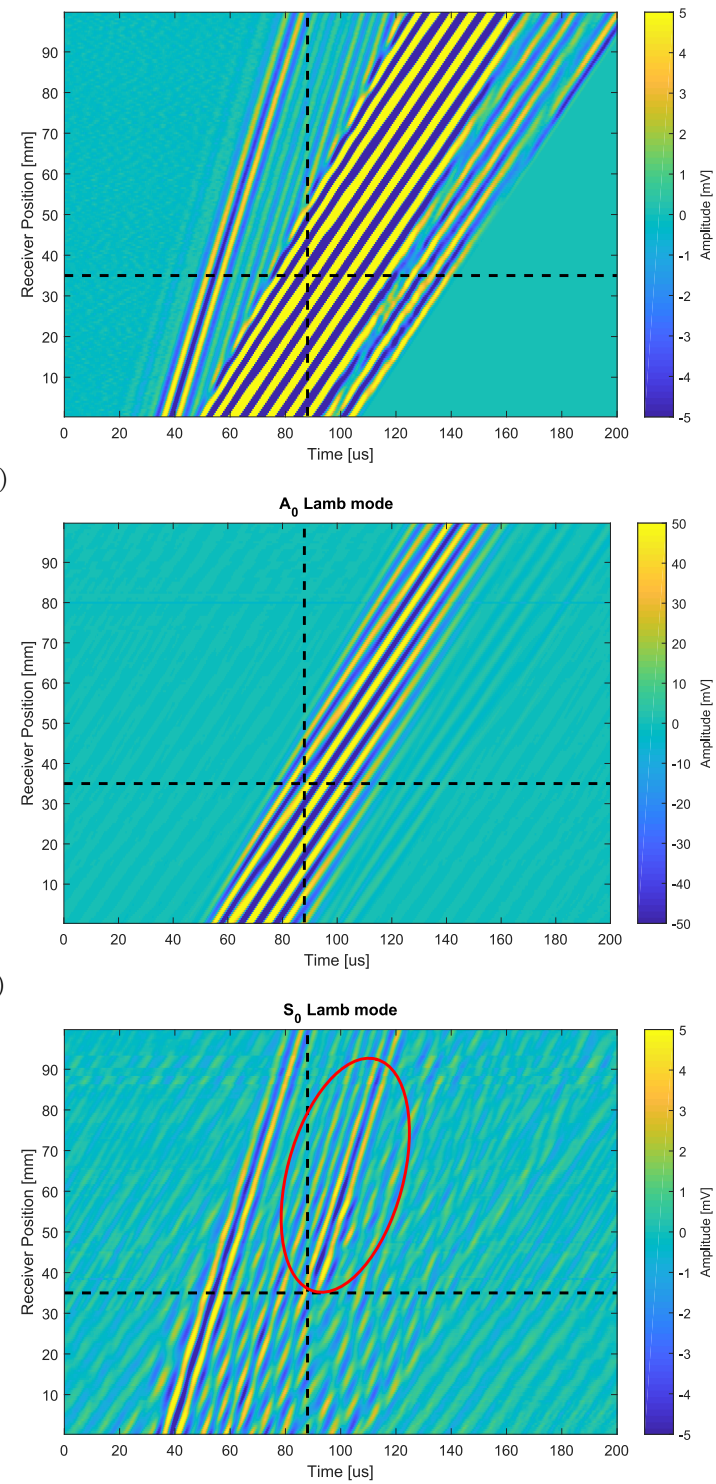

Figure 13: Lamb mode conversion. a) shows the B-scan of a plate with defect. b) shows the $A_{0}$ Lamb waves separated with the data driven signal analysis method, c) shows the $\mathrm{S}_{0}$ Lamb waves including the one that is mode converted from the $\mathrm{A}_{0}$ Lamb wave at the defect (red ellipse).

was run at every position with the data of the next 100 points. Therefore, some averaging effects occur around the defect position.

As one can see in the graph of the $\mathrm{S}_{0}$ Lamb mode 605 after the defect a new $S_{0}$ Lamb wave appears that is not present before the defect location. This $\mathrm{S}_{0}$ Lamb wave is mode converted at the defect location from the $\mathrm{A}_{0}$ Lamb mode. 


\section{Discussion}

Applying a window function to the original signal to separate the Lamb waves from the direct air signal will introduce errors no matter how it is done. The question may arise why the air signal is cut off at all. In theory, at least the data driven algorithm should be able to deal with this signal without further modifications and also the Lamb wave model could be extended by another (nondispersive) mode for sound in air. However, the measured data is clipped at $\pm 1 \mathrm{~V}$ to have enough amplitude resolution for the small Lamb wave signals. This leads to rectangular wave forms of the air signal and a lot of problems dealing with them.

The model based method could also be extended to other waves than plane waves by extending the model to also reflect a decay in amplitude. The data driven method does not allow other types than plane waves.

$\mathrm{Xu}$ et al 9] show mode conversion with a liquid coupled ultrasonic setup. Both, $A_{0}$ to $S_{0}$ and $S_{0}$ to

${ }_{630} \mathrm{~A}_{0}$ conversion occurs. In our case, no $\mathrm{S}_{0}$ to $\mathrm{A}_{0}$ can be noticed. The main reasons are the transmitter and receiver angles, which favor the $A_{0}$ to $S_{0}$ mode conversion by at least one order of magnitude.

The shape of the converted $\mathrm{S}_{0}$ mode is different

${ }_{635}$ than the one of the $\mathrm{A}_{0}$ mode it is originating from. One of the reasons for this behavior is the different out of plane displacement of the symmetric and anti-symmetric Lamb wave modes and also the receiver sensitivity for the $S_{0}$ mode. Additionally,

640 only a fraction of the energy of the incoming $A_{0}$ Lamb wave is converted to the $\mathrm{S}_{0}$ mode, leading to a lower signal amplitude.

In the time range where the setup allows to capture Lamb waves, the signal never decays to zero. Although the electrical excitation pulse is short (1.5 periods of a sine wave with $181 \mathrm{kHz}$ ), the excited pulse in the air has already gained additional width.

The data driven Lamb wave separation method yields good results that suffice to detect defects by mode conversion. Still, the $\mathrm{S}_{0}$ mode shown in Figure 13 seems to contain quite some amount of an $\mathrm{A}_{0}$ signal. A second pass through the algorithm may improve the results.

\section{Conclusion}

In this project we showed that in an air coupled ultrasonic setup multiple Lamb wave pulses are gen- erated in thin polymer plates, each containing one or more Lamb modes.

The most relevant parameters for the setup are the two distances transducer - plate surface $d_{S}$ and transmitter - receiver $d_{T x R x}$ and the transmitter angle $\alpha_{T x}$ as well as the receiver angle $\alpha_{R x}$. We demonstrated that it is favorable to mount the transducer close to the surface which leads to a longer window to detect Lamb waves but also to multiple reflections of the excitation pulse between transmitter and plate surface.

We showed the importance of a thorough data anal670 measured data and separate the different Lamb modes with a model based method and a data driven method. This also requires that the wave is measured at multiple locations along the propagation path.

Finally, as an application, we showed that Lamb mode conversion from the $\mathrm{A}_{0}$ mode to the $\mathrm{S}_{0}$ mode at the defect can be used for defect detection.

\section{Outlook}

This method can be extended to inspect a plate not only along one dimension, but also in two dimensions (2D-scan). One needs to deal with Lamb wave pulses that do not propagate exactly from the transmitter to the receiver, e.g. reflected modes at defects or object boundaries. These signals share the dispersion relation with the respective direct mode, but the different path of the waves may lead to a different wavenumber that is "seen" by the algorithms.

690 For defect detection, it is not absolutely necessary to move the receiver over the defect location. It is also possible to have the defect positioned somewhere between transmitter and receiver and to detect and localize the defect by backpropagation. It is also worthwhile to extend this inspection method to other types of material, e.g. composites. Two different things have to be considered for composites. First, different types of defects have to be included like delaminations and changes in stiffness. Second, the isotropy of the material parameters assumed in this paper may be violated in composites. Therefore the equations for waves in thin plates change as described by Rose [20]. Also the model in the model based separation algorithm needs to be capable of handling these parameters, whereas the data driven algorithm is not dependent on the material model. 


\section{References}

[1] D. Hutchins, D. Jansen, C. Edwards, Lamb-wave tomography using non-contact transduction, Ultrasonics 31 (2) (1993) 97-103 (1993).

[2] M. C. Bhardwaj, High transduction piezoelectric transducers and introduction of non-contact analysis, NDT.net 5 (1) (2000)

[3] J. Neuenschwander, R. Furrer, A. Roemmeler, Application of air-coupled ultrasonics for the characterization of polymer and polymer-matrix composite samples, Polymer Testing 56 (2016) 379-386 (2016).

[4] S. J. Sanabria, C. Mueller, J. Neuenschwander, P. Niemz, U. Sennhauser, Air-coupled ultrasound as an accurate and reproducible method for bonding assessment of glued timber, Wood science and technology 45 (4) (2011) 645-659 (2011).

[5] M. Castaings, P. Cawley, The generation, propagation, and detection of lamb waves in plates using air-coupled ultrasonic transducers, The Journal of the Acoustical Society of America 100 (5) (1996) 3070-3077 (nov 1996). doi:10.1121/1.417193

[6] C. Ramadas, K. Balasubramaniam, M. Joshi, C. Krishnamurthy, Interaction of guided lamb waves with an asymmetrically located delamination in a laminated composite plate, Smart Materials and Structures 19 (6) (2010) 065009 (2010).

[7] W. Ostachowicz, M. Radzieński, P. Kudela, M. Jurek, Non-contact excitation of guided waves using aircoupled ultrasonic transmitters for damage detection, in: Nondestructive Characterization and Monitoring of Advanced Materials, Aerospace, Civil Infrastructure, and Transportation XIII, Vol. 10971, International Society for Optics and Photonics, 2019, p. 109711J (2019).

[8] R. Kazys, A. Vilpisauskas, J. Sestoke, Application of air-coupled ultrasonic arrays for excitation of a slow antisymmetric lamb wave, Sensors 18 (8) (2018) 2636 (2018).

[9] K. Xu, D. Ta, Z. Su, W. Wang, Transmission analysis of ultrasonic lamb mode conversion in a plate with partial-thickness notch, Ultrasonics 54 (1) (2014) 395401 (2014).

[10] F. Benmeddour, S. Grondel, J. Assaad, E. Moulin, Study of the fundamental lamb modes interaction with asymmetrical discontinuities, NDT \& E International 41 (5) (2008) 330-340 (2008).

[11] F. Benmeddour, S. Grondel, J. Assaad, E. Moulin, Study of the fundamental lamb modes interaction with symmetrical notches, NDT \& E International 41 (1) (2008) 1-9 (2008).

[12] T. Hayashi, K. Kawashima, Single mode extraction from multiple modes of lamb wave and its application to defect detection, JSME International Journal Series A Solid Mechanics and Material Engineering 46 (4) (2003) 620-626 (2003)

[13] K. Xu, D. Ta, P. Moilanen, W. Wang, Mode separation of lamb waves based on dispersion compensation method, The Journal of the Acoustical Society of America 131 (4) (2012) 2714-2722 (2012).

14] A. Raghavan, C. E. Cesnik, Guided-wave signal processing using chirplet matching pursuits and mode correlation for structural health monitoring, Smart Materials and Structures 16 (2) (2007) 355 (2007).

15] J. Vollmann, R. Breu, J. Dual, High-resolution analysis of the complex wave spectrum in a cylindrical shel containing a viscoelastic medium. Part II. Experimental results versus theory, The Journal of the Acoustical Society of America 102 (2) (1997) 909-920 (1997).

[16] H. Lamb, On waves in an elastic plate, Proceedings of the Royal Society of London. Series A, Containing papers of a mathematical and physical character 93 (648) (1917) 114-128 (1917).

[17] J. L. Rose, Ultrasonic waves in solid media, Cambridge University Press, 1999 (1999).

[18] C. F. Tirendi, J. F. Martin, Quantitative analysis of nmr spectra by linear prediction and total least squares, Journal of Magnetic Resonance (1969) 85 (1) (1989) 162-169 (1989).

[19] A. Römmeler, R. Furrer, U. Sennhauser, B. Lübke, J. Wermelinger, A. de Agostini, J. Dual, P. Zolliker, J. Neuenschwander, Air coupled ultrasonic defect detection in polymer pipes, NDT \& E International 102 (2019) 244-253 (2019).

[20] J. L. Rose, Ultrasonic guided waves in solid media, Cambridge university press, 2014 (2014). 